\title{
THE BONE MICROSTRUCTURE IDENTIFICATION MODEL BASED ON BACKSCATTER MODE OF ULTRASOUND
}

\author{
Umiatin $^{1, *}$, Tora Oktaviana ${ }^{1}$, Endang Wijaya ${ }^{2}$, Riandini $^{2}$, Farhan Yusuf ${ }^{1}$ \\ ${ }^{I}$ Department of Physics, Universitas Negeri Jakarta, Rawamangun Muka, Jakarta, Indonesia. \\ ${ }^{2}$ Department of Electrical Engineering, Politeknik Negeri Jakarta, Depok, West Java, Indonesia \\ *Corresponding Author Email: umiatin@unj.ac.id
}

Received: 22 January 2021

Revised: 20 April 2021

Accepted: 22 April 2021

Online: 30 April 2021

Published: 30 April 2021

SPEKTRA: Jurnal Fisika dan Aplikasinya

p-ISSN: 2541-3384

e-ISSN: 2541-3392

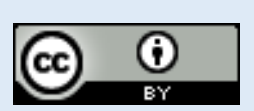

\begin{abstract}
Osteoporosis is defined by a decrease in bone mass and a deterioration in bone microstructure. It is a major public health issue and a significant economic burden for both individuals and society. Thus, monitoring bone mass and structure is necessary to prevent bone fragility and osteoporosis. This study aimed to develop a prototype of quantitative ultrasound (QUS) and to evaluate the feasibility of backscatter mode for the bone assessment. Ultrasound (US) signals that propagate through the bone can be characterized by comparing the signal from both transmitter and receiver transducers. The US backscattered signal depends on the characteristic of both medium and transducer. In this study, we analyzed the attenuated signal based on the parameters: type of bone (compact and spongy), type of coupling medium (air, starch, and gel), the angle between transducers and bone $\left(30^{\circ}, 60^{\circ}\right.$, and $\left.90^{\circ}\right)$, and transducer distance $(0,10,5,15,20$ and 25 $\mathrm{cm})$. We use only $1 \mathrm{MHz}$ transducer frequency. The prototype has been evaluated by Digital Oscilloscope and LabVIEW user interface to observe received signals. The results of this study showed that there was a difference in amplitude of the US signal from compact and spongy bones. The amplitude is directly proportional to acoustic impedance and inversely proportional to the distance between transducers. There is a negative correlation between bone microstructure to attenuation, and compact bones have a greater attenuation coefficient than spongy bones.
\end{abstract}

Keywords: osteoporosis, bone structure, ultrasound, backscatter, attenuation 


\section{INTRODUCTION}

Osteoporosis has become known as a silent disease. There is an increasing need to improve its diagnosis and management. Osteoporosis is currently a major public health problem suffered by millions of people around the world. Osteoporosis is a bone disease associated with decreased bone mass and microstructure as well as damage to bone tissue. Osteoporosis can lead to complications of fragility and the risk of fractures. The disease is closely related to aging factors, with a prevalence of more than $30 \%$ occurring in women over the age of 50 and in men of $15 \%$ [1]. The mortality rate due to osteoporosis and complications is about 15 to $30 \%$ [2].

About 200 million people have osteoporosis, and about 8.9 million suffer complications from fractures. This leads to a significant increase in morbidity, mortality, a decrease in quality of life, and an increasing economic burden [3]. In America, the economic burden for osteoporosis management reaches $\$ 13.8$ billion annually [4]. Data of osteoporosis risk conducted in 16 regions in Indonesia showed the total risk of osteopenia of $41.7 \%$ and osteoporosis of $10.3 \%$. The prevalence of bone and joint diseases based on the diagnosis of health workers in Indonesia is $11.9 \%$, with the highest prevalence in Bali being 19.3\%, followed by Aceh $18.3 \%$, West Java $17.5 \%$ Papua $15.4 \%$ [5].

The diagnosis of osteoporosis is generally done by evaluating Bone Mineral Density (BMD in gram/cm2) using DEXA (Dual Energy X-ray Absorptiometry). DEXA is used to determine the total mineral content of bone and bone mass density in the spine, hip, and arm bone. WHO approved DEXA as the gold standard for the diagnosis of osteoporosis. Based on WHO criteria, osteoporosis is defined as having a BMD between 2.5 standard deviations above or below the average value of a young healthy woman [6]. However, the technology used by DEXA is complex, result in DEXA operational costs are quite expensive [7]. The accessibility of DEXA equipment is also limited, only provided by type A hospitals that provide specialist medical services. Because of these limitations, several new methods for BMD assessment were studied as a prognosis tool in first-level health facilities. There is an urgent need for a portable, noninvasive, and radiation-free method to evaluate bone microstructure.

Quantitative Ultrasound (QUS) has been developed to evaluate bone mass density since 1984. This method uses sound waves with frequencies in the range $0.2-1 \mathrm{MHz}$ [8]. The sensitivity of QUS assessment of the calcaneus in the prediction of hip fracture has been shown by many studies. The value was similar to the mineral density of the hip bone measured by DEXA method [9]. Bone microstructure was quantified based on ultrasound speed values (speed of sound / SOS, m/s) and broadband ultrasound attenuation (BUA, db/MHz) [10]. This QUS device is considered a safe technology because it does not expose the patient to ionic radiation, unlike DEXA. Another advantage of QUS is that the units are smaller in size and lower operational costs, requiring no special space [11].

Several studies have reported the advantages and potency of an ultrasound method for the BMD evaluation. Lei and Jiping designed a prototype BMD measurement system by the Quantitative Ultrasond method based on FPGA (Field Programmable Gate Array). The results showed that the SOS and BUA values can reflect calcaneus bone conditions effectively, so the 
prototype can be used for a primary diagnosis of osteoporosis [12]. Furthermore, Wahab et al., 2016 designed an ultrasound-based bone densitometry system using FPGA. The circuit system was evaluated on the human arm and heel. At a distance of $10 \mathrm{~cm}$, the prototype provides a stable output voltage. The evaluation was also conducted by using many medium includes : water, gel, gel plus starch, and starch solutions. Water has a very small attenuation coefficient compared to other mediums. The A-mode ultrasound prototype was successfully characterized ultrasound signals through the human heel [13]. Umiatin et al. developed a QUS prototype with transmission mode using a $1 \mathrm{MHz}$ transducer frequency. The results showed that the BUA value in spongy bone is greater compared to compact bone [14].

Although QUS methods have been widely reported to assess bone density, the use in clinics is still limited and has not replaced the DEXA method. Therefore, further study is needed to improve the feasibility and safety of the QUS modalities. In this study, the development of the QUS method using backscatter mode has been carried out. The scattering signal from the bone sample was recorded for bone microstructure evaluation. These results have been compared with transmission mode in the previous publication.

\section{METHOD}

In this study, the QUS prototypes were developed with backscatter mode to evaluate the difference in BUA values between spongy and compact bones. The block diagram is shown in FIGURE 1.

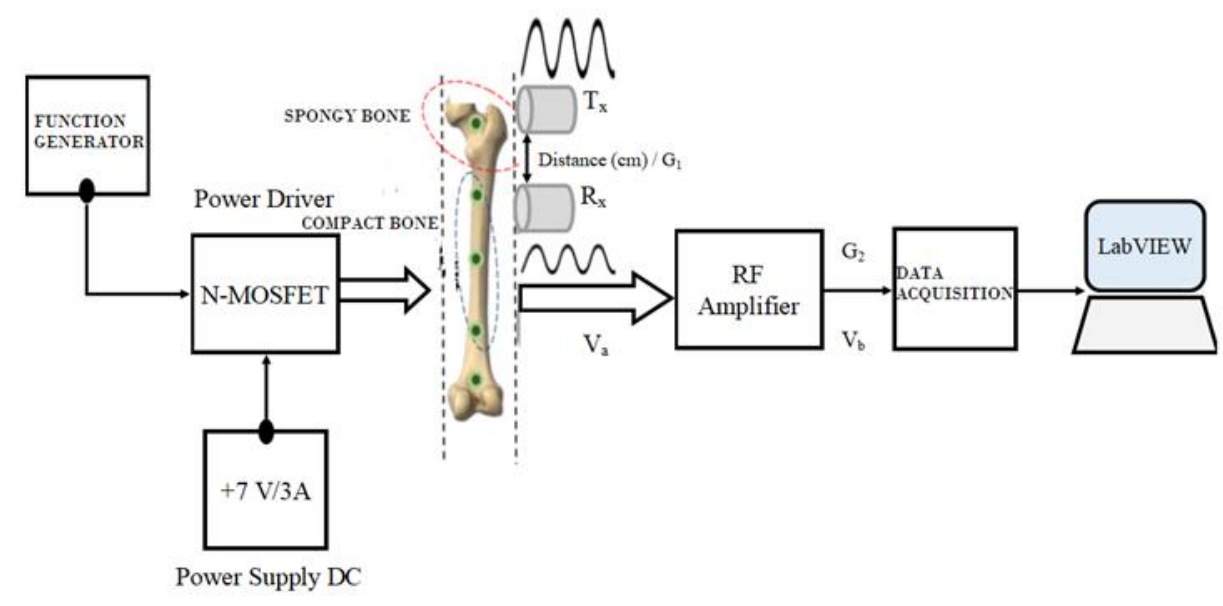

FIGURE 1. Block diagram of ultrasound quantitative system scattering mode

Function generators play a role in generating signals. The system consisted of two $20 \mathrm{~mm}$ diameter $1 \mathrm{MHz}$ ultrasound transducer, one acting as a transmitter the other as a receiver, and maintained at a fixed separation. Both transducers are placed parallel and have the same distance to the bones vertically. Tx transducers will convert electrical signals into ultrasound signals. The signal is passed to the bone that acts as a low pass filter to attenuate the amplitude. Furthermore, the signal received by the Rx transducer will be amplified by the amplifier. $\mathrm{Rx}$ signal display can be seen on the oscilloscope, and LabVIEW user interface as in FIGURE 2. 


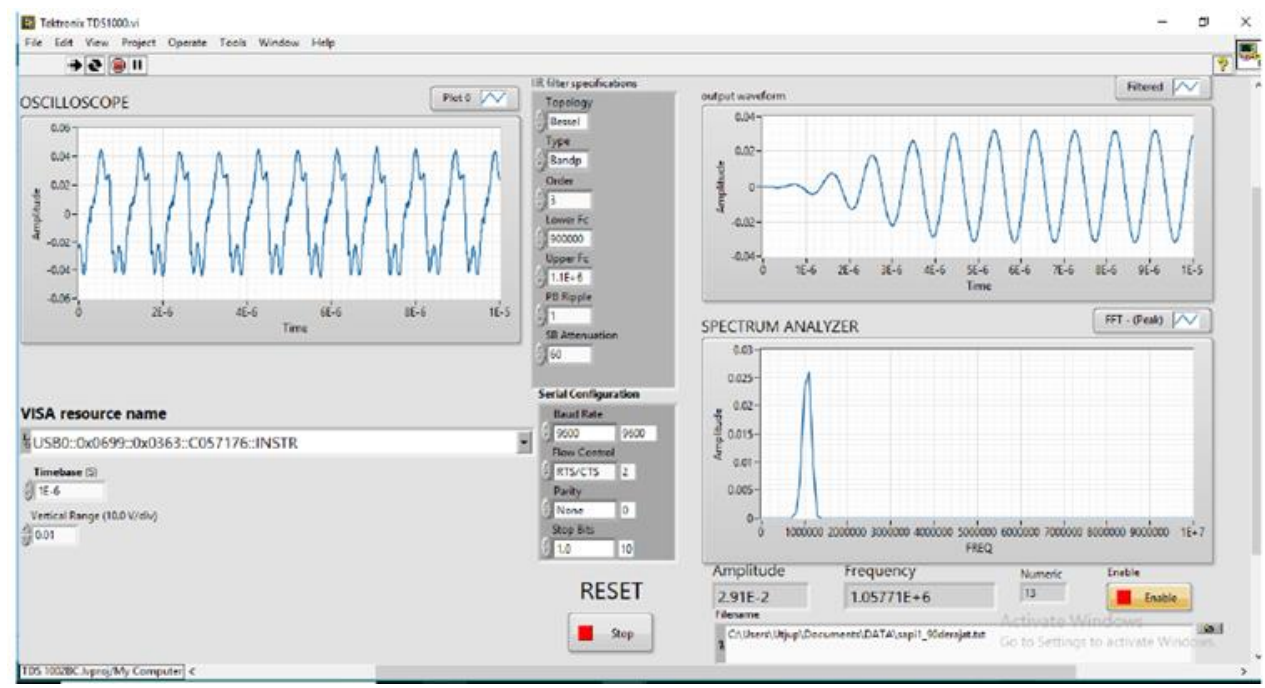

FIGURE 2. The user interface of the QUS prototype has been developed using the LabView program. It is consisting of initiating serial monitors, oscilloscope configurations, signal filters, output waveform graphs, and spectrum analyzer.

We have carried out measurements of ultrasound attenuation in the bone sample. The characteristic of ultrasound signal propagates through the bone depending on the type of bone, coupling medium, angle between transducer and bone, and transducer distance from bone. We used different angles between transducers with bone samples include $30^{\circ}, 60^{\circ}$, and $90^{\circ}$, as shown in FIGURE 3. The backscatter signal was highly variable depending on the angle of the transducer [17].
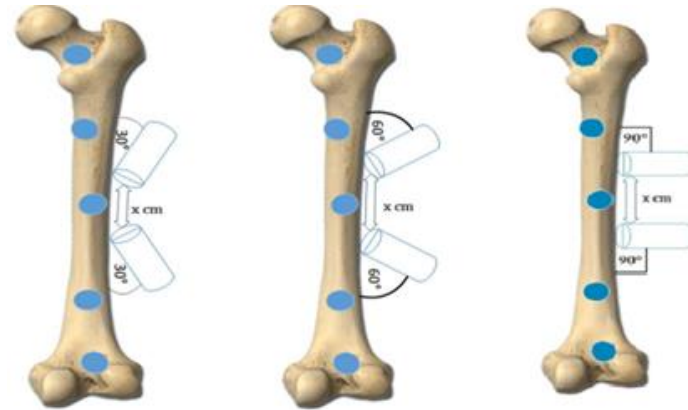

FIGURE 3. The angle variation between both transducers of Tx and Rx transducers to the bone include angles $30^{\circ}, 60^{\circ}$ dan $90^{\circ}$.

In this study, we also performed backscatter ultrasound signals from different transducer distances to the bone samples, involve: $0,5,10,15$, and $20 \mathrm{~cm}$. The coupling medium used consists of air starch and gel. Then, the attenuated ultrasound signal from the receiver was analyzed to compare the bone microstructure.

\section{RESULTS AND DISCUSSION}

The complex architecture of bones and the contribution of organic and inorganic material produce a very strong bio-structure. Commonly, the clinical osteoporosis diagnostic modalities such as DEXA [16]. Ultrasound modalities are a low-cost, non-ionizing, and 
portable method that correlates with BMD and the potential to assess the bone structure and its mechanical properties. The most commonly used parameters: the broadband ultrasound attenuation (BUA) and the speed of sound (SOS) in the transmission mode, are good indicators of the bone condition. Ultrasound backscatter parameters based on heterogeneous material scattering theory have proven useful for evaluating the microstructure of spongy bone [15]. Scattering means that an ultrasound signal when it hit an obstacle, irradiates some of its energy in all spatial directions. The backscattered signal can be detected through the well-known pulse-echo technique [17].

When the ultrasound signal passes through the medium with different acoustic properties, then there will be a change in amplitude. The signal propagation is depend on the characteristics of the bone sample as the medium. The signals attenuate mainly due to reflection, absorption and scattering, resulting in a decrease in amplitude and intensity. The Rx transducer receives attenuated signals with varying intensities and amplitude depending on many factors. In this study, we evaluated factors that involve: the coupling medium, the bone type, and the angle between transducers and sample, and the frequency of transducers.

\section{Optimization of The Distance Between Transducer and Bone}

In this study, the distance between bones and both transducers, Tx and Rx were changed. In the first measurement, the distance between Tx and Rx was optimized with bone samples and air medium. A distance of $0 \mathrm{~cm}$ is the distance of a transducer that is squeezed with arm bones with a thickness of $3.6 \mathrm{~cm}$, which means a distance of Free Space Loss (FSL) of $0 \mathrm{~cm}$. Data retrieval was conducted as many as 10 experiments every 30 seconds, as shown in FIGURE 4.

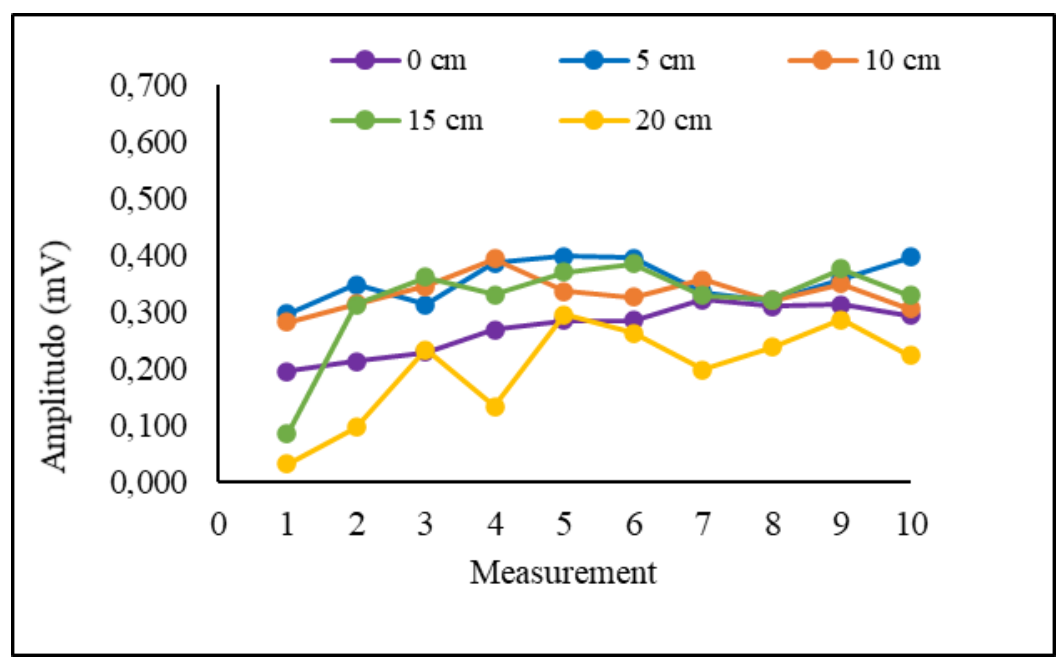

FIGURE 4. Transducer distance optimization to bone samples

High and stable voltage results are obtained at a distance of $5 \mathrm{~cm}$. Thus, measurement using other conduction mediums, gel, and starch, will be conducted at a fixed distance of $5 \mathrm{~cm}$. The output voltage is affected by the distance between the transducer and the medium. The gap between the two transducers is called FSL or gain (G1). When the ultrasound signal leaves the 
transmitter, the signal will propagate in the air so that there is a weakening of the signal and the value of G1 becomes negative. However, the signal is re-amplified by a positive value amplifier (G2) to pass to the receiver.

\section{Optimization of Coupling Medium}

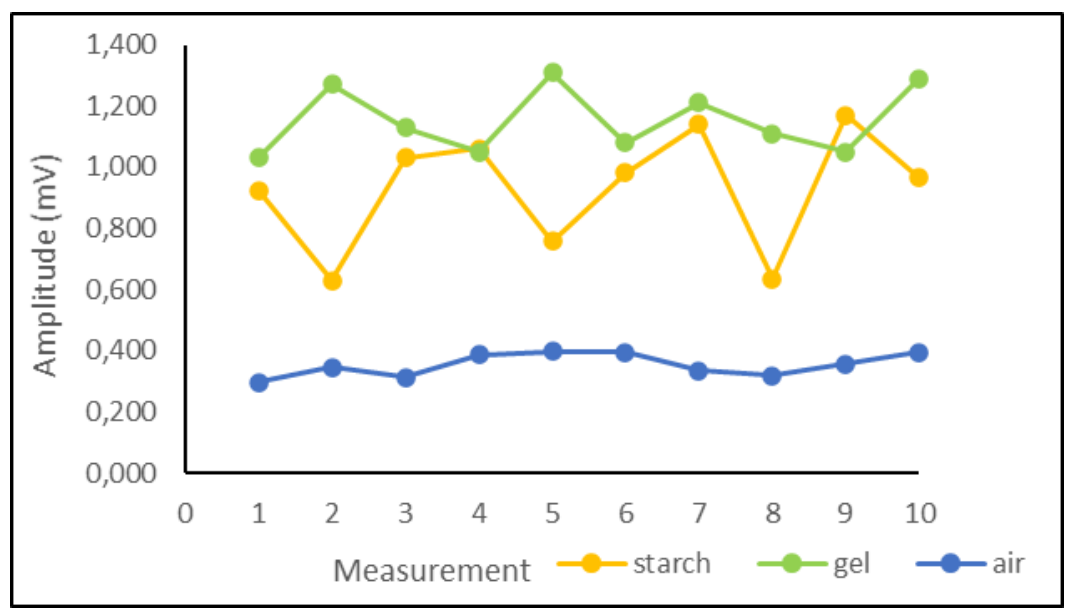

FIGURE 5. Optimization of coupling medium includes: air, starch, and gel.

FIGURE 5 showed that the gel medium has a higher amplitude voltage than the air and starch. The average amplitude are $0.35 \mathrm{mV}, 1.15 \mathrm{mV}$, and $1.28 \mathrm{mV}$ in air, starch, and gel. It can be concluded that the attenuation in the gel is lower than in both of air and starch. The gel medium can reduce reflection and improve the transmission of ultrasound signals to the bone.

The acoustic impedance of air and one has a considerable difference, which is about $4,310^{2}$ $\mathrm{kg} / \mathrm{m}^{2} \mathrm{~s}$ in air and $6,010^{6} \mathrm{~kg} / \mathrm{m}^{2} \mathrm{~s}$ in bone. Based on the calculations, $99.97 \%$ of the ultrasound signal will be reflected by the bone. The excessive reflection ratio will make it difficult to identify bone microstructure because the ultrasound signal received by the receiver will be released into the air. To reduce the reflection, the gel medium is used. The composition of the gel is mostly water, so the impedance of the gel can be considered equal to water $1,4810^{6}$ $\mathrm{kg} / \mathrm{m}^{2} \mathrm{~s}$. The level of ultrasound reflection by using the gel as a coupling medium is about $36.51 \%$. This is in contrast to research conducted by Wahab et al. [13], the best transmission medium of ultrasonic waves is water.

\section{Optimization of The Angles between Transducers and Bone Samples}

In this study, the optimization of the angle between the transducer and the bone was carried out. The angle variations used are $30^{\circ}, 60^{\circ}$, and $90^{\circ}$ with a constant Tx and Rx distance of 5 $\mathrm{cm}$. Cow femur bones are evaluated at five different sites, namely, points $1-5$, as shown in FIGURE 6. 


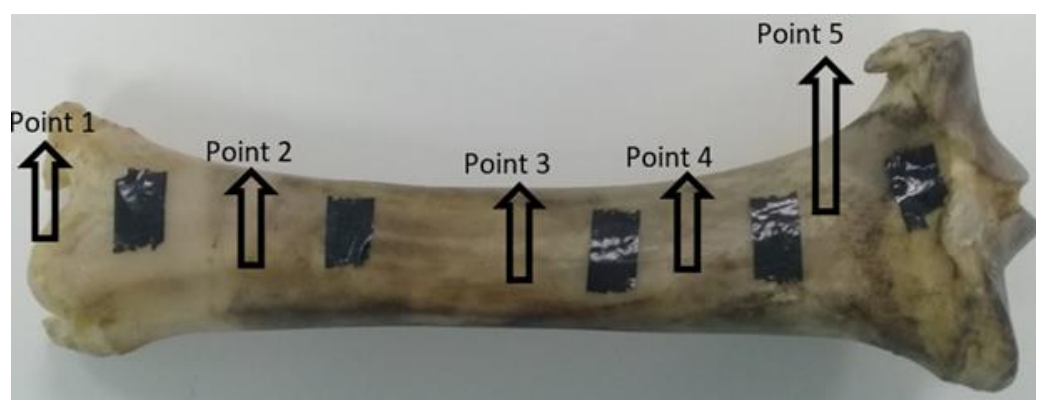

FIGURE 6. Femur bone evaluation site. The thickness of each area : point $1(4.49 \mathrm{~cm})$, point $2(3.21 \mathrm{~cm})$, point $3(3.79 \mathrm{~cm})$, point $4(4.94 \mathrm{~cm})$, point $5(6,36 \mathrm{~cm})$.

FIGURE 7 shows the microstructure of each point recorded using the digital microscope with a 50 times magnification.

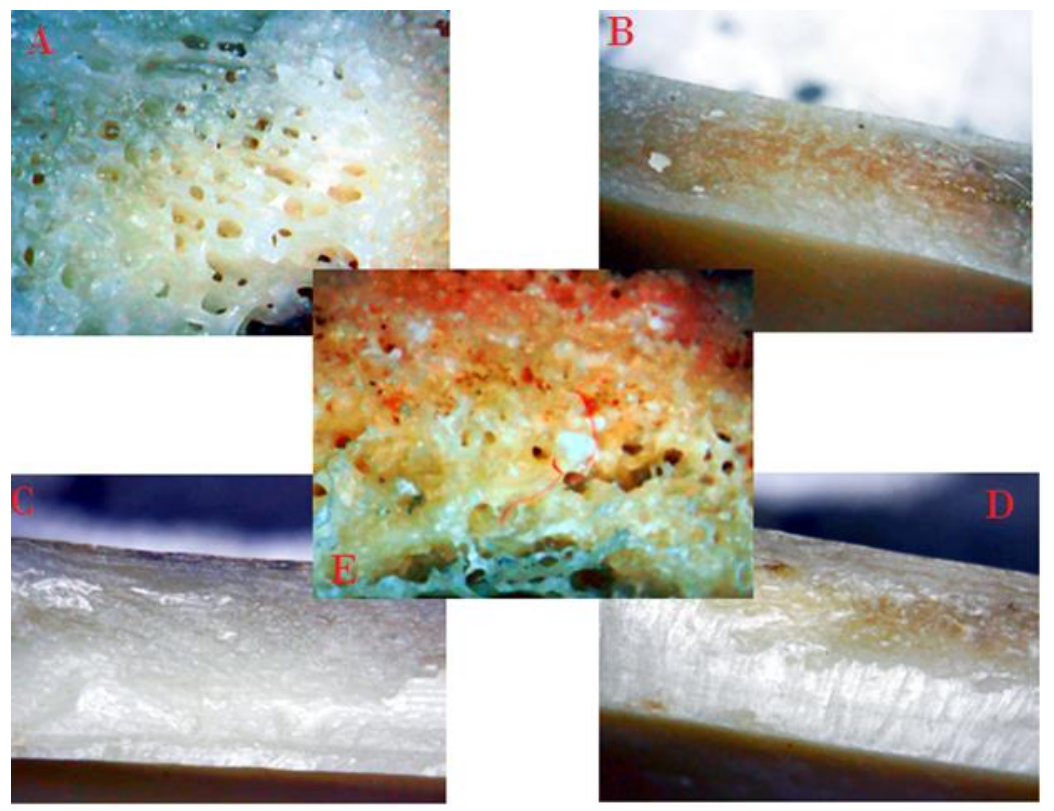

FIGURE 7. The microstructure of the bone sample is divided into two categories: spongy bone (A, E) and compact bone.

Femur bone test results with a frequency of $1 \mathrm{MHz}$ at three angular variations with medium gel are shown in FIGURE 8 and 9. 


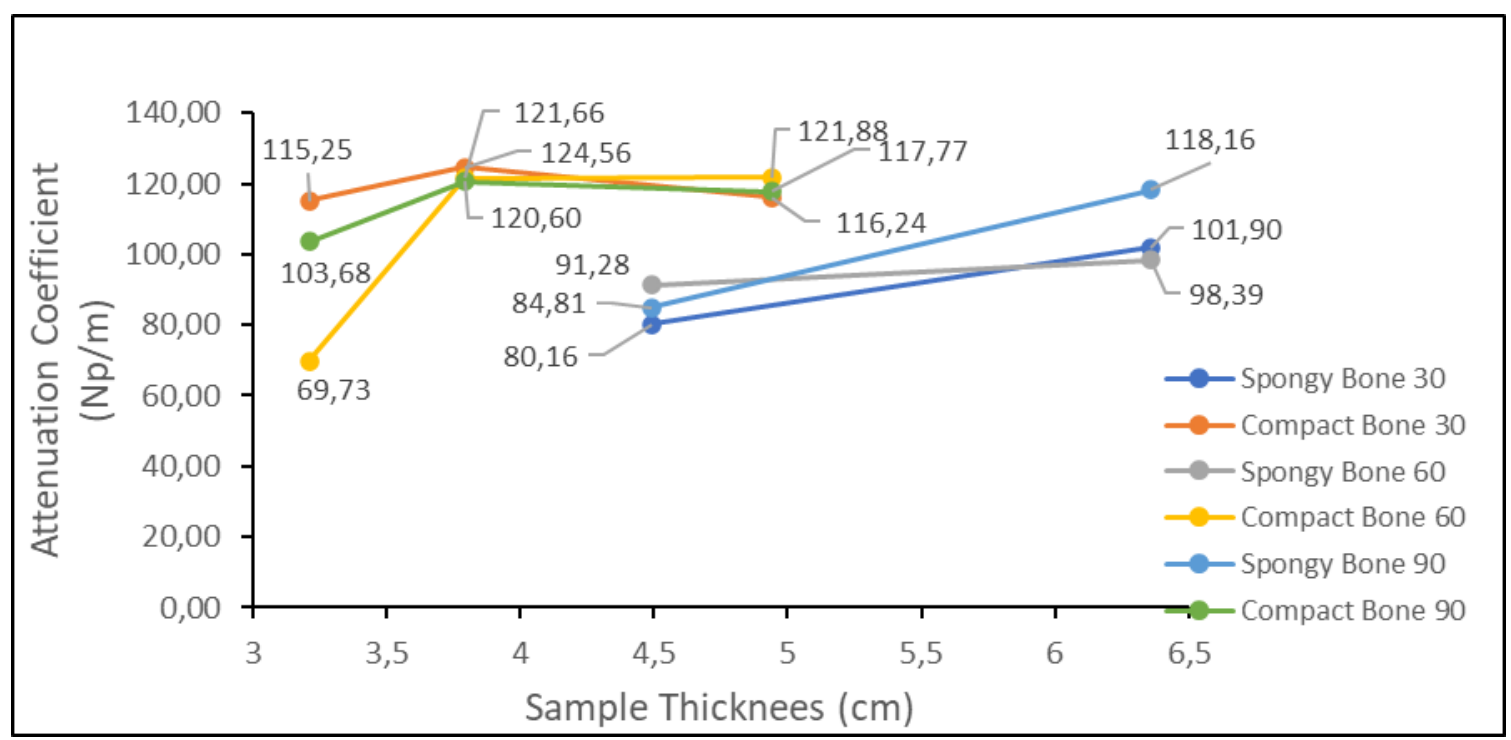

FIGURE 8. Comparison between US attenuation value at $30^{\circ}$ transducer angle of the compact and spongy bone sample.

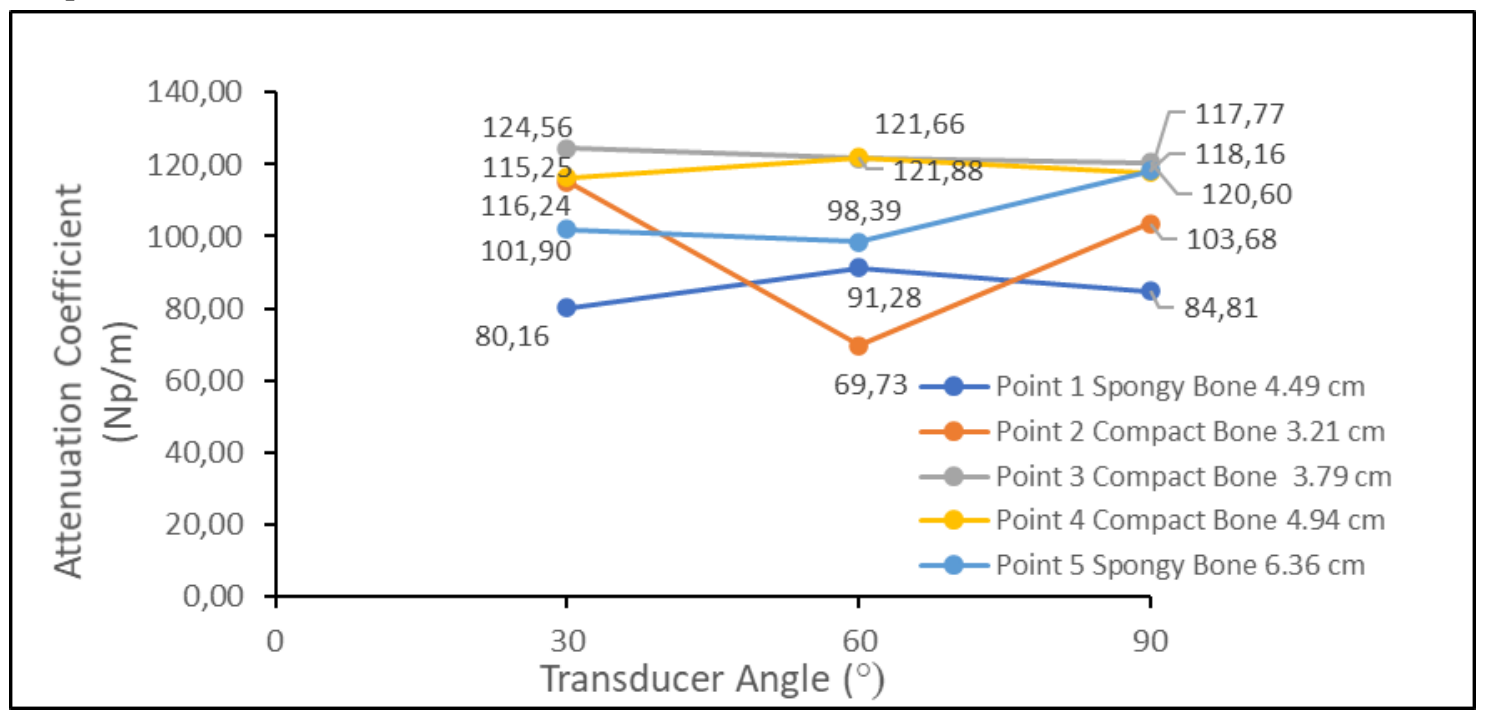

FIGURE 9. Comparison between US attenuation value at $30^{\circ}$ transducer angle of the compact and spongy bone sample.

FIGURE 8 and 9 show that the largest attenuation level is on point 3 at an angle of $30^{\circ}$ by 124.56 Nepres $/ \mathrm{m}$. The smallest attenuation level is on point 1 at an angle of $30^{\circ}$ by 80.16 Nepres $/ \mathrm{m}$. The largest coefficient of attenuation indicates a greater density in compact bone. The smallest attenuation coefficient occurs in the compact bone at an angle $60^{\circ}$ by 69.73 Nepres/m. Overall, at each transducer angle, the attenuation coefficient of spongy bone is smaller than that of compact bone. The attenuation value also depends on the thickness of the bone samples. Compared to the results in the previous publication, where the position and direction of the two transducers face each other against the bone sample, the backscatter method not better than the transmission method [14]. However, Iori et al. reported that the backscatter mode could be used to predict the size of compact bone, which is a major factor 
in bone strength [18]. The limitation in this study only uses $1 \mathrm{MHz}$ working transducer frequency. Therefore the variation in the BUA dependence frequency can not be analyzed.

\section{CONCLUSION}

This article presents the backscatter mode of Quantitative Ultrasound that can be used to identify bone structures based on differences in attenuation coefficients. The results showed that the smaller angle between the transducer and the sample, the greater coefficient of attenuation. The largest attenuation of the femur bone is obtained in compact bone with a transducer angle of $30^{\circ}$ of $124.56 \mathrm{~Np} / \mathrm{m}$ of the lowest attenuation with a transducer angle of $60^{\circ}$ of $80.16 \mathrm{~Np} / \mathrm{m}$ on compact bones.

\section{REFERENCES}

[1] A. Rashki Kemmak et al., "Economic burden of osteoporosis in the world: A systematic review,” Med J Islam Repub Iran, vol. 34, p. 154, 2020, doi: 10.34171/mjiri.34.154.

[2] T. A. G. Nevin Hammam, "Epidemiology and awareness of osteoporosis: a viewpoint from the Middle East and North Africa," Int. J. Clin. Rheumatol, 2018, doi: 10.4172/1758-4272.1000177.

[3] S. Minisola et al., "New anabolic therapies for osteoporosis," Intern Emerg Med, vol. 12, no. 7, pp. 915-921, Oct. 2017, doi: 10.1007/s11739-017-1719-4.

[4] E. Hernlund et al., "Osteoporosis in the European Union: medical management, epidemiology and economic burden," A report prepared in collaboration with the International Osteoporosis Foundation (IOF) and the European Federation of Pharmaceutical Industry Associations (EFPIA), Arch Osteoporos, vol. 8, p. 136, 2013, doi: 10.1007/s11657-013-0136-1.

[5] “Laporan-Riskesdas-2018-Nasional.pdf,” Accessed: Apr. 07, 2021. [Online]. Available: https://dinkes.kalbarprov.go.id/wp-content/uploads/2019/03/Laporan-Riskesdas-2018Nasional.pdf.

[6] I. Akkawi and H. Zmerly, "Osteoporosis: Current Concepts," Joints, vol. 6, no. 2, pp. 122-127, Jun. 2018, doi: 10.1055/s-0038-1660790.

[7] C. Hartman et al., "Assessment of Osteoporosis by Quantitative Ultrasound versus Dual Energy X-Ray Absorptiometry in Children with Chronic Rheumatic Diseases," The Journal of Rheumatology, vol. 31, no. 5, pp. 981-985, 2004.

[8] C. M. Langton, S. B. Palmer and R. W. Porter, "The measurement of broadband ultrasound attenuation in cancellous bone," Eng Med, vol. 13, no. 2, pp. 89-91, Apr. 1984, doi: 10.1243/emed_jour_1984_013_022_02.

[9] C. F. Njeh, C. M. Boivin and C. M. Langton, "The role of ultrasound in the assessment of osteoporosis: a review," Osteoporos Int, vol. 7, no. 1, pp. 7-22, 1997, doi: 10.1007/BF01623454.

[10] D. Hans and M. A. Krieg, "Quantitative ultrasound for the detection and management of osteoporosis," Salud pública Méx, vol. 51, pp. s25-s37, 2009, doi: 10.1590/S003636342009000700006. 
[11] D. Hans and S. Baim, "Quantitative Ultrasound (QUS) in the Management of Osteoporosis and Assessment of Fracture Risk," J Clin Densitom, vol. 20, no. 3, pp. 322333, Sep. 2017, doi: 10.1016/j.jocd.2017.06.018.

[12] Y. Lei and Y. Jiping, "Design of BMD measurement system based on FPGA," Aug. 2012, pp. 1385-1388, doi: 10.1109/ICICEE.2012.366.

[13] M. A. A. Wahab et al., "Design of an A-mode ultrasound amplifier for bone porosity detection," in International Symposium on Electronics and Smart Devices (ISESD), pp. 79-84, Nov. 2016, doi: 10.1109/ISESD.2016.7886696.

[14] Umiatin et al., "Design of bone density identification method using transmission quantitative ultrasound,” AIP Conference Proceedings, vol. 2169, no. 1, p. 030012, Nov. 2019, doi: 10.1063/1.5132662.

[15] W. Mao et al., "Ultrasound Backscatter Technique for Assessing and Monitoring Neonatal Cancellous Bone Status in Vivo," IEEE Access, vol. 7, pp. 157417-157426, 2019, doi: 10.1109/ACCESS.2019.2949748.

[16] B. Lashkari, L. Yang, and A. Mandelis, "The application of backscattered ultrasound and photoacoustic signals for assessment of bone collagen and mineral contents," Quant Imaging Med Surg, vol. 5, no. 1, pp. 46-56, Feb. 2015, doi: 10.3978/j.issn.22234292.2014.11.11.

[17] C. Liu et al., "The relationship between ultrasound backscatter and trabecular anisotropic microstructure in cancellous bone," Journal of Applied Physics, vol. 115, no. 6, p. 064906, Feb. 2014, doi: 10.1063/1.4865173.

[18] G. Iori et al., "Estimation of Cortical Bone Microstructure From Ultrasound Backscatter," IEEE Transactions on Ultrasounds, Ferroelectrics, and Frequency Control, vol. 68, no. 4, pp. 1081-1095, Apr. 2021, doi: 10.1109/TUFFC.2020.3033050. 